\title{
Characterization of 3D Dopant Distribution in State of the Art FinFET Structures
}

\author{
M.S. Hatzistergos ${ }^{1}$, M. Hopstaken ${ }^{2}$, E. Kim ${ }^{3}$, L. Vanamurthy ${ }^{3}$ and J.F. Shaffer ${ }^{1}$ \\ ${ }^{1 .}$ IBM Systems and Technology Group, Hopewell Junction, NY 12533, USA \\ 2. IBM T.J. Watson Research Center, Yorktown Heights, NY 10598, USA \\ ${ }^{3}$ GLOBALFOUNDRIES, Albany Nanotech Center, Albany, NY 12203, USA
}

Fin field-effect transistors (FinFETs) are a promising alternative for next generation CMOS technology [1]. A significant structural difference from the long-established planar FETs is the three dimensionality aspect of the device. The schematic illustration in Figure 1 depicts the main structural differences between a planar FET and a FinFET.

Dopant characterization in the semiconductor industry is traditionally performed using Secondary Ion Mass Spectrometry (SIMS). Dedicated SIMS-pads with appropriate dimensions (100's of micrometers) have been used over the years to characterize dopant distributions. A large planar pad that has been exposed to the same processing steps as the planar device is analyzed and the doping levels on the actual device are derived. Planar pads can no longer be used, as effectively, to characterize dopant distributions in a fin. Therefore, Atom Probe Tomography (APT), that provides three dimensional (3D) compositional characterization with sub-nanometer resolution and detection limits of the order of $100 \mathrm{ppm}$, is expected to play a significant role in the development of FinFETs. In APT a sample, shaped into a cone with a tip curvature of the order of 50nm, is evaporated and later reconstructed atom by atom in 3D [2]. The prepared tips were analyzed by a Cameca FlexTAP. A FEI Helios Nanolab 400 dual beam was used following standard APT sample preparation procedures [3].

Knowledge of FinFET doping profiles is essential. Conformal fin sidewall doping on the source/drain regions is necessary to minimize access resistance. SIMS depth profiles take into account hundreds of fins, provide average top down information with good detection limits and can be used to monitor a process. APT has a limited field of view (typically of the order of 100nm) and therefore the results may not be representative. However, the sub-nanometer resolution provides dopant positioning information in 3D and when necessary can be used for site specific characterization and failure analysis.

In this work, large arrays of FinFETs representative of $14 \mathrm{~nm}$ CMOS technology were analyzed. Fins were patterned on bulk Si wafers and were exposed to a source/drain plasma doping process. After a wet clean step, the trenches between the fins were filled with poly-Si and the wafers were planarized by CMP in order to prepare the surface for SIMS and APT. Such large array of fins allows the use of the "SIMS through fins" approach and simplifies the APT sample preparation since there is no need to target a specific area (sub-100nm fin pitch ensures the presence of 2-3 fins on a given APT tip) [4].

Three samples were analyzed with the following characteristics: as doped, cleaned and Argon knock-on plus clean. The wet clean process is expected to remove surface doping and reduce the fin dopant concentration, while the Argon knock-on process is expected to drive into the fin dopants and increase dopant retention after the subsequent wet clean. Figure 2 depicts top down profiles acquired by SIMS and APT with general agreement between the two techniques, however additional improvements in APT Boron detection efficiency and reconstruction algorithms are necessary [5]. Boron concentration is significantly reduced after the wet clean step and the Ar knock-on process has helped with dopant 
retention but only on the top part of the fin due to shadowing effects. Due to the high spatial resolution capabilities of APT, unique horizontal (through the fin sidewalls) profiles are extracted in addition to individual fin top down profiles. Figure 3 illustrates a set of such plots from the "as doped" specimen.

\section{References}

[1] C.-H. Lin et al,VLSI 2012.

[2] T.F. Kelly and M.K. Miller, Rev. Sci. Instrum, 78(3), 031101 (2007).

[3] K. Thompson et al, Ultramicroscopy 107(2-3), 131-139 (2007).

[4] J. Mody et al, JVSTB 28(1) C1H5 (2010).

[5] P. Ronsheim et al, Applied Surface Science 255, 1547-1550 (2008).

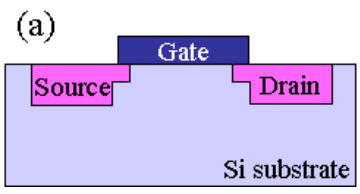

(b)

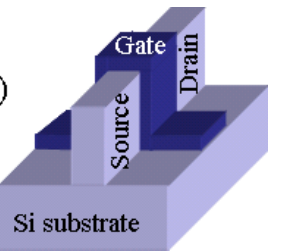

Figure 1. (a) Traditional planar device with a gate controlling the on/off state. (b) FinFET, a doped vertical fin with a gate wrapped around it.
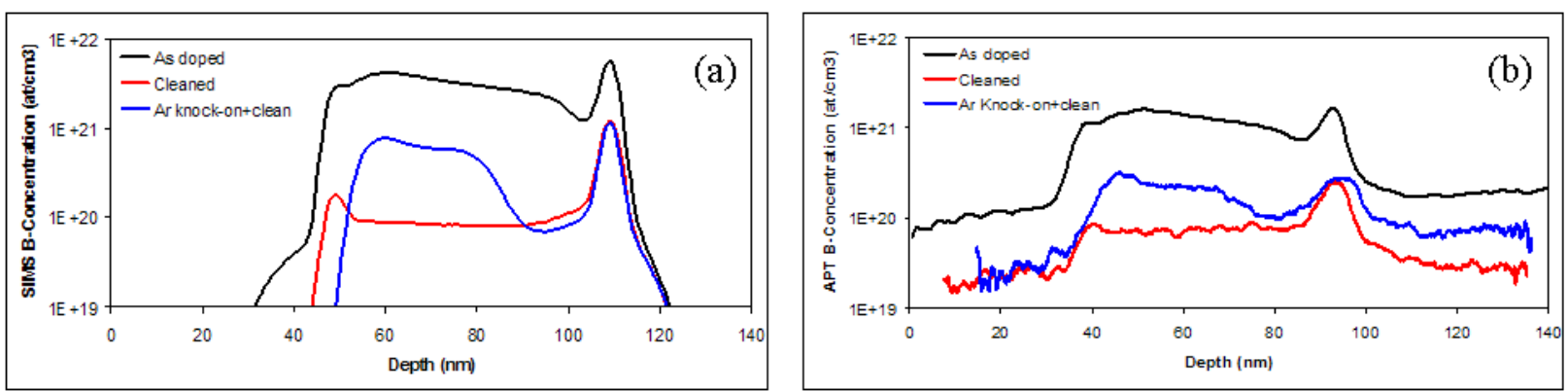

Figure 2. Top down profiles: (a) SIMS and (b) APT (average of 2-3 fins).
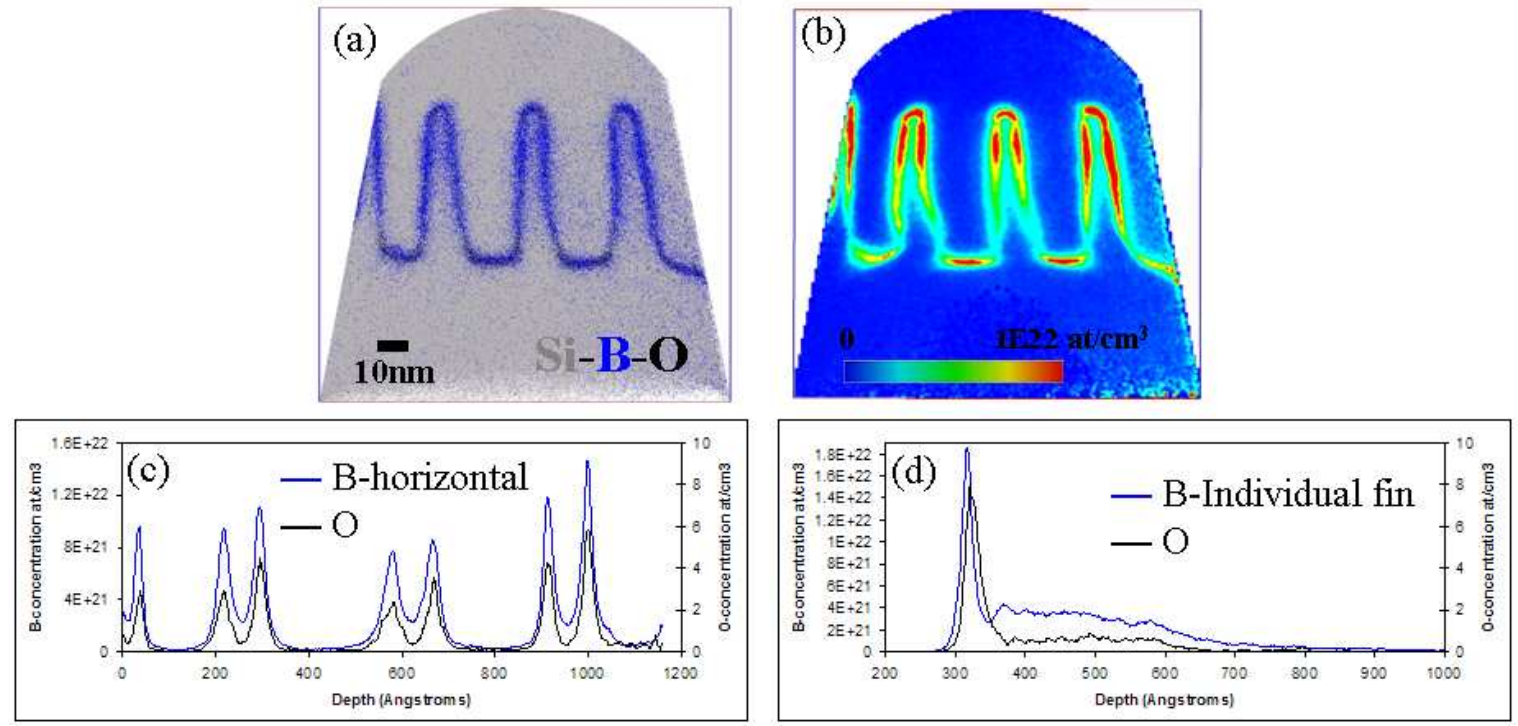

Figure 3. (a) Atom map depicting Silicon, Boron and Oxygen atoms, (b) 2D Boron map, (c) horizontal (through the fin sidewalls) $\mathrm{B}$ and $\mathrm{O}$ profiles and (d) individual fin $\mathrm{B}$ and $\mathrm{O}$ top down profiles. 